\title{
A study on the current practices of software development in Malaysia
}

\begin{abstract}
Software development process and software quality have important roles in government and education sector as our society nowadays becomes more dependent on computers technology especially in system or software product development. Thus, the effectiveness of project management is an important factor to find out the achievement of the software development processes as well as level of software quality. Previously, a research studies regarding the practices of software development process have been conducted by many researchers to examine the effectiveness of the practices including research studies were conducted in European countries, India, Japan and just a few in Asian countries especially in Malaysia. With regard of this situation, this research study conduct a survey in order to investigate current practices of software development process activities as well as identifies the methodology, programming languages and techniques used in software development processes. The structured questionnaire have been distributed to the respective respondents for data collection followed by data extracting, data analyzing, evaluation and discussion of the results. Basic statistical methods which are frequency and cross tabulations were used to analyze the data. The results of this research study have addressed some important points regarding the practices of software development processes used in government and education sectors in Malaysia such as gives impact towards a better quality of software or system. Furthermore, implementing of project management process are not in maximum approach which show that project management are not fully implemented in development of a project.
\end{abstract}

Keyword: Software project management; Software development process; Software product 